\title{
Investigation of Porous Silicon Photoconductive Structures
}

\author{
Petro Parandiy \\ Dept. of Electronics and Computer Technologies \\ Ivan Franko National University of L'viv \\ L'viv, Ukraine \\ e-mail: petro.parandiy@lnu.edu.ua
}

\author{
Liubomyr Monastyrskii \\ Dept. of Electronics and Computer Technologies \\ Ivan Franko National University of L'viv \\ L’viv, Ukraine
}

\begin{abstract}
Porous silicon layers photoconductive and electrical properties obtained by electrochemical etching of silicon have been investigated. Photoconductive and currentvoltage properties depend on structure morphology are determined by not only properties of modified layer, but presence of charge carriers' traps. The photosensitivity of structures with PS layers is determined by thickness of porous layer.
\end{abstract}

Index Terms-Porous silicon; sensor; photoconductivity; current-voltage characteristic; photosensitivity

\section{INTRODUCTION}

At a present time, the problem of controlling toxic and other harmful substances in the air atmosphere, drinking water, foodstuffs, etc. expected immediate solving. Thereby, there is a necessity for the development of microelectronic gas detection systems for industrial, office and residential apartment, which can be components of integral systems for protecting human life and health. Sensors based on nanoporous semiconductors, in particular, porous silicon (PS) are attracting special attention.

A set of optical sensors are described in a number of scientific papers [1-6]. Among such sensors we can separate photosensitive structures on porous silicon, based on the change of photoconductivity and its kinetics under the influence of various gas media, which can be used as effective gas analyzers. To design such sensors, it is necessary to know the dependences of the porous silicon photoconductivity on the state of the surface, which depends on the gas environment in which it is located, as well as the degree of the material porosity, the pores geometry and the frequency of modulation of the incident light intensity.

The porous silicon (PS) is predisposing the attention of researchers mostly due to its luminescent properties [2]. That is, since as in the layers of the PS was detected photoluminescence (PL) with a high quantum efficient at room temperature, this material became the object of intense both fundamental and applied research. Such a widely broad study of various properties of the PS has opened prospects for its numerous alternative applications in areas such as solar cells, biotechnology, sensors [1,8-9].

PS is a promising material for silicon electronics to creating gas and liquid sensors through a combination of crystalline structures and the giant external surface (200-500 $\mathrm{m}^{2} / \mathrm{cm}^{3}$ ) [3-4]. Effect of adsorption phenomena on the photoconductivity and current-voltage characteristics of PS structures are at the stage of initial studies with following practical application, based on the fact that the sensors are analytical tools for the analysis of solutions thanks to such characteristics as the express analysis, the ability of miniaturization and automation, simplicity their use and low cost.

Results of adsorption influence on electrophysic properties occur in the heterostructures formed on porous silicon based on silicon substrates of different types of conductivity under reaction of environmental hydrogen containing molecules (acetone $\left(\mathrm{CH}_{3}\right)_{2} \mathrm{CO}$, toluene (methylbenzene) $\mathrm{C}_{6} \mathrm{H}_{5}$ $\mathrm{CH}_{3} \mathrm{C}_{2} \mathrm{H}_{5} \mathrm{OH}$, benzene $\mathrm{C}_{6} \mathrm{H}_{6}$ ) are presented.

Gas molecules adsorption leads to the surface photoconductors recombination speed enhancing by increasing the charge carriers capture on energy trap levels is caused by adsorbed atoms, as well as the change in the structure of $\mathrm{Si}-\mathrm{Si}$ "dangling" bonds. The intensity of photoconductors recombination at small gas concentrations is proportional to the partial pressure of the adsorbed exhaust and depends on its dipole moment. Molecules of adsorbate with a large dipole moment amplify the capture of electrons (holes) at the trap energy levels, which leads to an increase in the rate of recombination through these levels and changes the magnitude of photoconductivity. Thus, the effect of changing the photoconductivity of porous silicon under the influence of adsorbed gases is promising for the creation of effective gas sensors.

\section{EXPERIMENT}

\section{A. Porous Silicon Forming}

To produce samples by electrochemical etching a set of single crystal silicon wafers obtained by zone melting were used with crystallographic orientation (100) and (111). PS layers were grown directly on silicon substrates of $p$ - and ntype doping with a resistivity of 0.01 to $10 \Omega \cdot \mathrm{cm}$.

PS layers growing carried out by electrochemical anodizing silicon crystal substrates in electrolytes based on hydrofluoric acid (HF). Ethanol solution of $\mathrm{HF}$ better 
penetrate inside the pores, which is very important for the horizontal layer uniformity in depth of PS crystal.

For electrophysical investigation the set of PS electrochemically etched samples were selected PS-nSi (ntype silicon doped sulfur substrate marked KES-0,01 and KES-4.5, $\mathrm{j}=35 \mathrm{~mA} / \mathrm{cm}^{2}, \mathrm{t}=50 \mathrm{~min}$ ).

Etched substrates were separated on the single samples of $1 \mathrm{~cm}^{2}$ square approximately. For establishing conductive properties of PS the three-point contact scheme was applied. Contact to the porous layer and back side about $2 \mathrm{~mm}$ in diameter was obtained by electrically conductive graphite varnish or glue. Current-voltage curves were obtained by PS surface in planar mode.

\section{B. Experimental Approaching}

Measuring cell for all kind of PS properties investigations was constructed with possibility of input-output complicated vapor and gaseous mixture and external irradiation.

Current-voltage characteristics (CVC) of samples were measured at gases and vapours dose environment and in their absence. Time of measurement was about 5 minutes. All measurements were provided in the planar mode (on the surface of porous samples) on automatic equipment based on micro device controlling with capability of programming control. The set of experimental curves were measured by applying on the structure constant external bias supply at $15-+15 \mathrm{~V}$ with a rate $100 \mathrm{mV} / \mathrm{s}$, sampling interval was $0.2 \mathrm{~s}$, save interval was $0.2 \mathrm{~s}$. Current range was set in auto ranging mode as $10 \mathrm{nA}-10 \mathrm{~mA}$. Signal was fixed under normal conditions in a parallel circuit measurement. For eliminating the influence of light, measurements were carried out in the dark.

For research porous silicon sensor structures dependencies, we used the measuring device E7-22 RLC-meter, voltmeter V7-21A, bridge resistances. Electric supply was applied from a source of constant tension B5-45. As voltmeter and ampermeter used combined digital multimeters.

For obtaining of PS photoconductivity spectral dependencies, the sample was contained in the measuring cell without substrate heating, and all measurements were carried out at room temperature and under normal environmental conditions. The main PS photoconductivity investigation scheme a diffraction monochromator, amplifier, light source, registering device.

The modulating system is applying for photosensitivity investigations. All photosensitive spectral dependencies were conducted on the spectrophotometric measuring installation based MDR-12. Lightning system consist of light source, condenser system, spectral device, mechanical modulator. A 100W Tungsten halogen lamp integrated with convex ellipsoidal mirror with maximum at the near IR was used as the photon beam source.

\section{POROUS SILICON SURFACE MORPHOLOGY}

The study of the PS sample surface morphology was carried out a scanning electron microscope REEMA-102-02 and on scanning probe microscope SolverNEXT with nanoindenter Solver NexT, NT-MDT, by atomic force microscopy (AFM) technique at the semi-contact mode in the air.

Fig. 1 presents the plan view SEM micrographs of PS prepared on the substrate of n-type conductivity KES-0.01 (PS-nSi), obtained with a scanning electron microscope REEMA-102-02. Flat crystalline silicon substrate surface look like mirror due to the manufacturing polishing. PS layer after electrochemical anodization in the plan-view the sponge-like islands macroarray of 200-200 nm sizes is clearly observed. Color surface after electrochemical etching is dark with yellow contrast. This form of PS surface has such pore shape depends on orientation of silicon wafer and the way of electrochemical dissolution. Cross-section scan electron image confirm that the PS layer has sponge-shaped disordered etched pores. The thickness for porous orientation was about $1 \mu \mathrm{m}$.

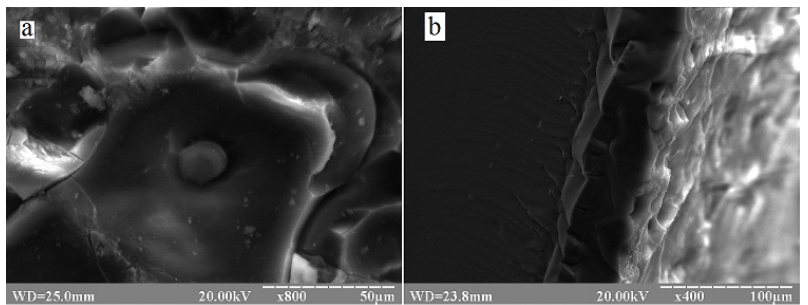

Fig. 1. a) PS-nSi scanning electron surface image, b) PS-nSi cross-section scanning electron surface image.

Fig. 2 shows the surface of the PS on the substrate of ntype conductivity KEF-4.5 (PS-nSi), obtained with a scanning electron microscope REEMA-102-02. PS is matte and dark and there is clearly square-like macroarray structure surface with quadrate network of 5-10 nm size. Color surface after electrochemical etching is dark. At a first look, the PS surface seems to be not to etched to form porous layer. But we observe the tendency of silicon nanowires forming among crystallographic axes of silicon wafer. The characteristic faceted, square shaped pores illustrate an orientation dependence of the photo anodization process of the Si (111) material, probably due to the anisotropy of $\mathrm{Si}$ (111) dissolution. The thickness for porous (111) layer was around $0.5 \mu \mathrm{m}$.

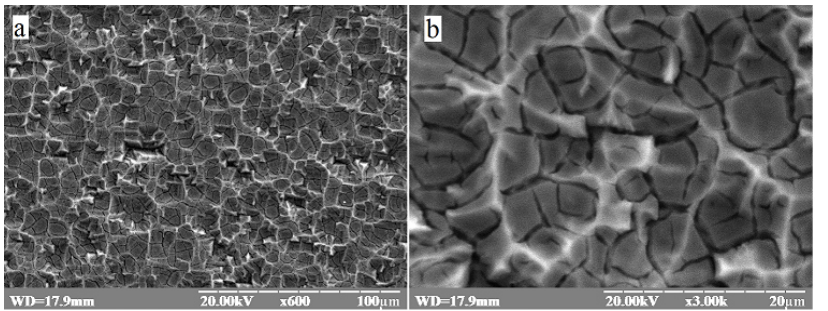

Fig. 2. a) PS-nSi (111) scanning electron surface image (600*), b) PS-nSi 111) scanning electron surface image $(3000 *)$.

PS sample surface morphology was carried on scanning probe microscope. The image, seen on fig. 3 obtained by atomic force microscopy, give possibility to observe squarelike structure with forming an island of etched silicon substrate. Explanation of such porous surface growth tendency is caused by a huge etching time with electrolyte heating and surface penetrating. 


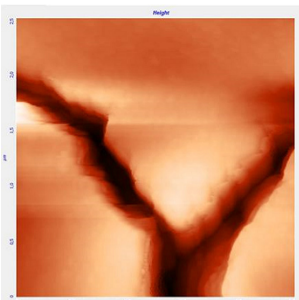

a

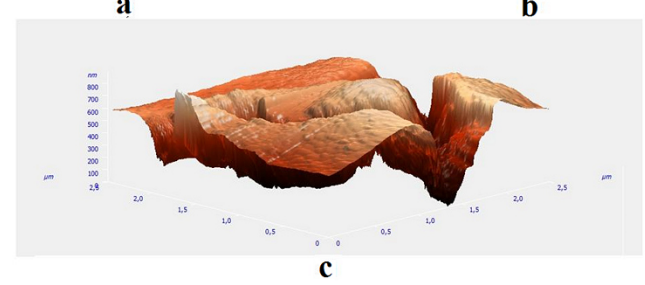

Fig. 3. PS-nSi scanning probe microscope image in next regimes: a) 2Dtopography; b) phase contrast, c) 3D-image

Analysis of PS surface studies of etched samples and comparison with literature data, lead to the conclusion that the origin of cracks, islands, protrusions and cells on the PS surface is due to the emergence and formation of silicon ledge formed on the surface of crystalline silicon under the PS layer of the material of the silicon substrate during the etching.

\section{Porous SiLICON STRUCTURE PHOTOCONDUCTIVITY}

Fig. 4 represent dynamic PS-nSi current-voltage curves, measured to establish an effective range for photoconductivity registration. The structure of current-voltage curves has a rectifying character, and there is no saturation of either direct or reverse current. The reverse current increases with increasing bias, and the direct is extrapolated by exponential dependence.

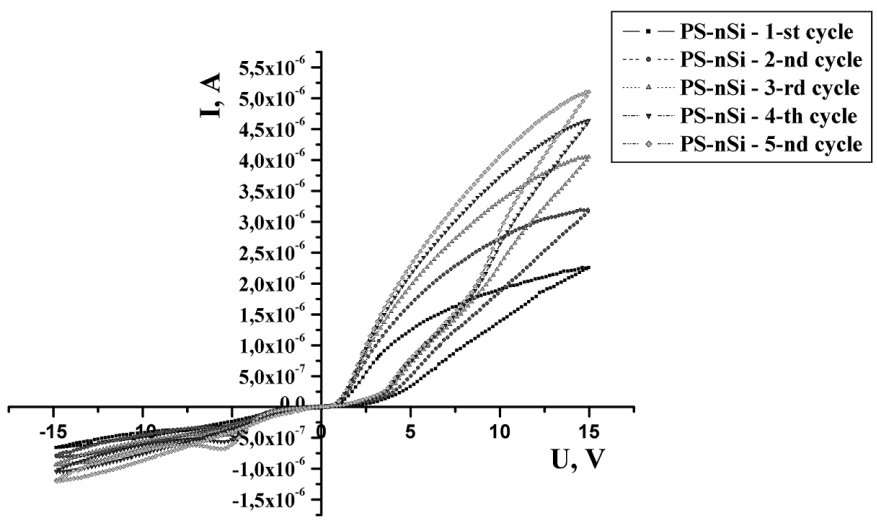

Fig. 4. PS-nSi structure dynamic current-voltage characteristic

Hysteresis loops were observed on dynamic currentvoltage characteristic, measured with a low frequency step, indicating that the holes were captured on the traps (Fig. 10, b). The typical times of direct and reverse current setting at pulse shift were respectively 40 and $13 \mathrm{~s}$. This indicates a presence of slow traps $[11,12]$.

Fig. 5 shows the time dependencies of the current output with the applied external bias of $+5 \mathrm{~V}$.

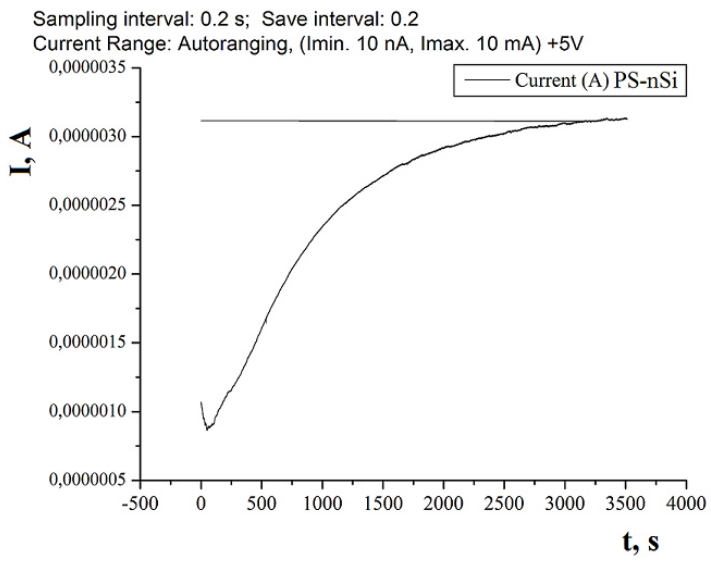

Fig. 5. Current output time dependencies under external bias $+5 \mathrm{~V}$.

As can be seen in fig. 5, under natural light and under normal atmospheric conditions, the time dependences of the current output at the applied external tension of $+5 \mathrm{~V}$ indicate a considerable time to reach a stable state of electrons fulfilling due external tension and the instability of the spectral dependences of the photoconductivity.

Fig. 6 shows the spectral dependence of the PS-nSi photoconductivity with a thickness of about $0.5 \mu \mathrm{m}$, where the sample was oriented toward the light porous surface. Investigation of the photoelectric properties of PS layers on the silicon substrate is complicated by the fact that the absorbed radiation penetrates the PS layers into the silicon wafer.

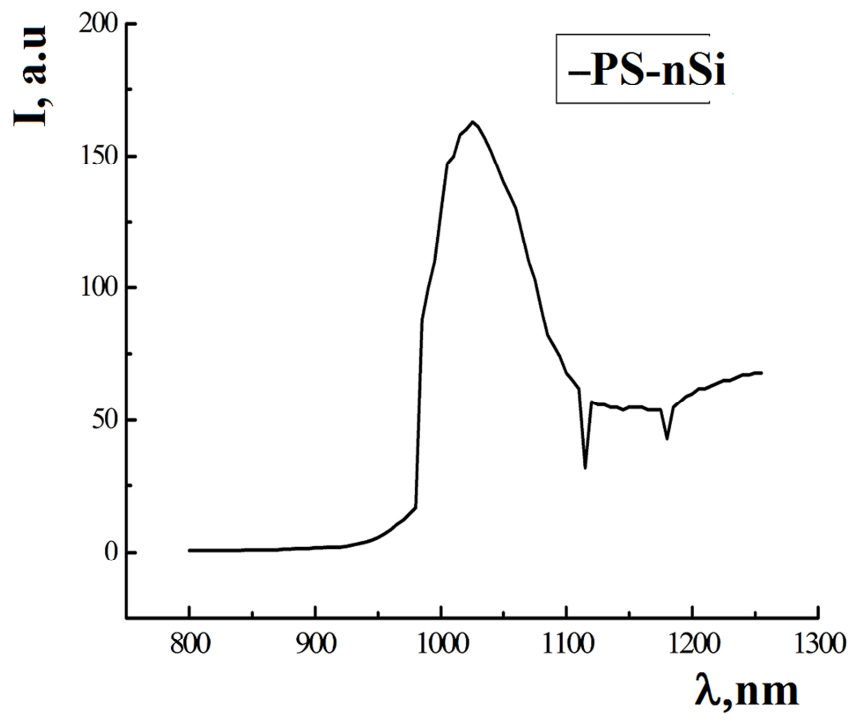

Fig. 6. PS-nSi photoconductivity at the room temperature $(\mathrm{U}=+8 \mathrm{~V})$

At the value of the applied voltage $+8 \mathrm{~V}$, the spectrum of the photoconductivity has a maximum current output of approximately the value of the applied voltage of order $10^{-10} \mathrm{~A}$ with the precision to the applied bias. However, in the photoconduction spectrum, flash flares of charge relaxation are observed at $+8 \mathrm{~V}$ in the direction of reduction of current. The maximum is at $1045 \mathrm{~nm}$, the region of spectral dependence 800-1200 $\mathrm{nm}$. 
Such photoconductive properties can be explained by processes of charge carrier generation under the influence of light. In the case of studying the n-doped silicon substrate, the carriers generated in the PS are recombined near the boundary of the charge spatial division region. The direction of the current depends on the value of the applied voltage. This affects the depth of light penetration into the PS-nSi structure and the depths of the levels where the carriers originate. Radiation with less energy results in photoconductive generation at deeper levels and before the partition of carriers on the PS-nSi barrier, depending on the applied field. The PS is transparent to light - the sensitivity spectrum is associated with generation charges in $\mathrm{Si}$, not $\mathrm{PS}$, and their recombination at the boundary of the PS-nSi. The light passes into the PS layer, in a wide geometric range. This light releases charges from traps of the Si substrate levels, and it plays a key role in the flow of current.

The presence of thick layers of PS on silicon substrate type KES-0.01 showed the presence of a change in the current conductivity signal only in the visible area of 500-800 nm. One of the peculiarity is the presence of a spectral dependence of conductivity in the region with a maximum at $650 \mathrm{~nm}$.
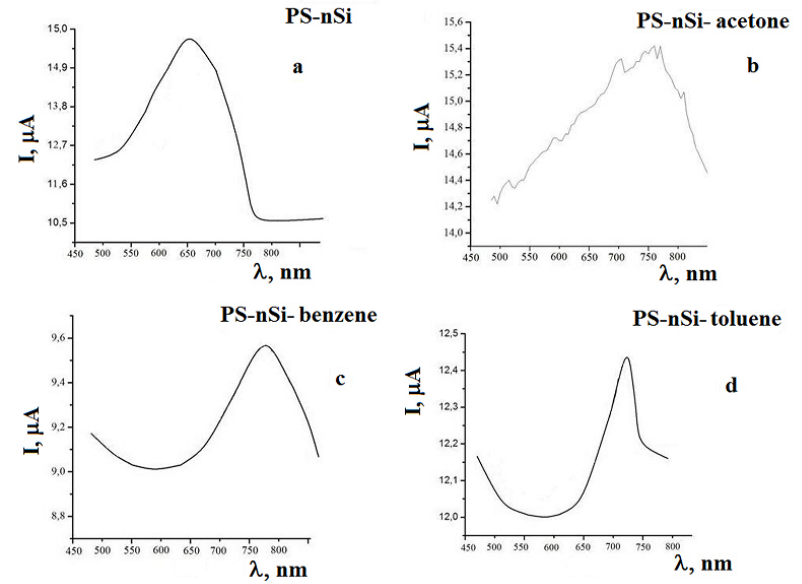

Fig. 7. PS photoconductivity at room temperature: a) normal atmospheric conditions; b) atmosphere of acetone vapor; c) atmosphere of benzene vapor; d) atmosphere of toluene vapors.

Therefore, an attempt was made the measurement of conductivity spectral dependence at the presence of harmful hydrocarbon compounds in the vapor environment. Fig. 7, b shows the spectra of the photoconductivity for PS acetone vapor content of $3.17 \mathrm{ppm}$. There is a shift in the maximum sensitivity for small signals for the test sample in the region of $760 \mathrm{~nm}$. This can be explained by the adsorption of acetone molecules on the PS surface. At the presence of benzene vapor (2.61 ppm), reduction of conductivity can be explained by passivation of PC surface 'dangling' bonds by benzene rings, which leads to the appearance of additional traps on the surface of the investigated structure. Toluene evaporation (3 $\mathrm{ppm}$ ) in a closed air volume resulted in a shift in the maximum band of the photoconductivity to a region of $730 \mathrm{~nm}$ and a decrease in the current output with the probability of passivation of the surface bonds of the PS. In addition, the toluene molecules act as acceptors. This explains the decrease in photoconductivity.

\section{ACKNOWLEDGMENT}

Due to the photosensitivity of porous silicon surface based on morphology and current-voltage characteristics, the investigated object can be applied as a gas sensor of harmful environmental gas due to the complicated and branched surface of the investigated structure. photoconductive properties can be explained by processes of charge carrier generation under the influence of light. The presence of harmful hydrocarbon compounds in the vapor environment with definite content is leading to a shifting of the sensitivity maximum. This can be explained by the adsorption and influence of polarized hydrocarbon molecules on the PS surface properties.

\section{REFERENCES}

[1] T.I. Gorbanyuk, A.A. Evtukh, V.G. Litovchenko, V.S. Solnsev, E.M. Pakhlov, "Adsorption of hydrogen containing molecules in the multilayers structures based on the porous silicon and palladium films," Physics And Chemistry Of Solid State, V. 7, № 1 (2006) P. 60-66.

[2] Rittersma Z. M., "A monitoring instrument with capacitive porous silicon humidity sensors," Smart Mater. Struct., 2000, Vol. 9, p. 351356.

[3] C. Baratto, G. Faglia, G. Sberveglieri, "Multiparametric porous silicon sensors," Sensors, 2002, vol. 2, p. 121-126.

[4] O. I. Ksenofontova, A. V. Vasin, V. V. Egorov, A. V. Bobyl', F. Yu. Soldatenkov, E. I. Terukov, V. P. Ulin, N. V. Ulin, O. I. Kiselev, "Porous silicon and its applications in biology and medicine," Technical Physics, January 2014, Volume 59, Issue 1, pp. 66-77.

[5] N. L. Dmitruk, O. S. Kondratenko, L. A. Vlasukova, P. V. Kuchynsky, "Optical and sensitive properties of amorphous $\mathrm{SiO} 2$ tin dioxide with embedded into ion tracks on silicon sensor," Electronics and Microsystem Technologies, 2013, v. 10, № 1, pp. 40-46.

[6] L. Monastyrskii, R. Yaremyk, I. Olenych, P. Parandii, "Multielement sensory systems based on porous silicon," Visnyk of the Lviv University, Series Physics, 2011, Issue 46, pp. 189-195.

[7] Canham L.T. Appl. Phys. Lett., vol. 57, 1990, p.1046-1048.

[8] Cullis, AG., Canham, LT. \& Calcott, PDJ, "The structural and luminescence properties of porous silicon,” J. App. Phys., 1997, vol. 82, No 3, pp. 909-965.

[9] Canham L., "Properties of porous silicon," INSPEC - The Institution of ElectricalEngineers, 1997, United Kingdom.

[10] Foll, H., Christophersen, M., Carstensen, J., Hasse, G., "Formation and application of porous silicon," Materials Science and Engineering R, 2002, Vol. 39, pp. 93.

[11] Foll, H., Christophersen, M., Carstensen, J., Hasse, G., "Formation and application of porous silicon," Materials Science and Engineering R, 2002, Vol. 39, pp. 93.

[12] K. Kulathuraan, K. Mohanraj, B. Natarajan, "Structural, optical and electrical characterization of nanostructured porous silicon: Effect of current density," Spectrochimica Acta Part A: Molecular and Biomolecular Spectroscopy, 152, 2016, pp. 51-57. 